\title{
Classification of Haploid and Diploid Maize Seeds based on Pre-Trained Convolutional Neural Networks
}

\author{
Emrah Dönmez ${ }^{1 *}$ \\ ${ }^{1}$ İnönü University, Central Campus Battalgazi/Malatya, Turkey \\ *emrah.donmez@inonu.edu.tr \\ *Orcid No: 0000-0003-3345-8344
}

Received: 26 May 2020

Accepted: 14 September 2020

DOI: 10.18466/cbayarfbe. 742889

\begin{abstract}
Analysis of agricultural products is an important area that is widely emphasized today. In this context, with the development of technology, computer-aided analysis systems are also being developed. In this study, a system has been proposed for classifying maize seeds as haploid and diploid using pre-trained convolutional neural networks. For this purpose, AlexNet, GoogLeNet, ResNet-18, ResNet-50, and VGG16 pre-trained models have been used as feature extractors for the haploid and diploid seed classification process. In the first stage, the deep features of haploid and diploid maize seeds have been obtained in these models. The features have been taken from different layers of network architecture. Instead of softmax classifier in the last layer of the network, classifiers based on decision tree, k-nearest neighbor, and support vector machine have been used. According to the classification results with these features, the achievements in network architectures and classifier methods have been observed. The experiments have been carried out on a publicly available dataset consisting of 3000 haploid and diploid maize seed images. The experimental results revealed that the developed classification systems demonstrate a remarkable performance.
\end{abstract}

Keywords: Haploid Maize Seed Identification, Deep Features, Artificial Learning, Convolutional Neural Networks, Image Processing

\section{Introduction}

The researches have focused on computer-aided agriculture systems in terms of providing advantages such as growing healthy products, increasing production efficiency, and reducing costs in recent years. The main contribution of these systems is that they save time and labor. Computer-aided agricultural researches have gradually gained momentum $[1,2]$. These systems are utilized extensively in most of the agricultural processes. The agricultural tools supported by these systems as well are commonly developed today. These systems prevent product havoc caused by human errors and ensure maximum efficiency.

In vivo maternal haploid breeding method has become the standard method in modern maize breeding because of its advantages compared to traditional methods [3]. In this maize breeding method, artificial chromosome folding is performed by treating haploid individuals with single chromosomes with colchillin and $100 \%$ pure lines can be obtained in 1-2 years [4]. Haploids must be separated from diploids after hybridization in order to increase the effectiveness of the method and to be successful. Depending on the characteristics of the inducer line used, haploid individuals in the range of 2$15 \%$ are obtained [5]. Separation of haploid individuals during the seed period is of great importance in terms of reducing greenhouse area, labor, and planting costs [4].

Although different genetic markers have been proposed to differentiate haploid maize seeds from diploids, the most common and successful method is the R1-nj color marker. In this method, which has been first used by Nanda and Chase [6], the inducer line carries the dominant R1-nj gene, which causes red-purple coloration in the embryo and endosperm. Since diploid seeds obtained as a result of crossbreeding carry chromosomes belonging to the inducer line, this coloration occurs in both embryos and endosperms. Haploid seeds, on the other hand, carry the chromosome of the inducer line in their endosperms, their embryos with single $n$ chromosomes consist only of the chromosomes of the donor parent. Therefore, R1-nj coloration is seen in the endosperms of haploid seeds, but R1-nj coloration does not occur in their embryos. 
This coloration difference occurring in embryos enables the visual separation of haploid and diploid seeds [7]. This separation is done manually today. In addition to this, it causes separation with high error rates; costs increase and separation takes a long time. Computeraided classification methods are developed to overcome these problems. Today, with the advances in machine learning methods, deep learning methods based on convolutional neural networks $(\mathrm{CNN})$ are widely used for computer-aided classification studies.

$\mathrm{CNN}$ aims to calculate the features of an object in image data based on multiple processing layers of artificial neural networks without using an external method [8]. Image processing techniques are used for the detection of the features (color, shape, texture, pattern, etc.) of the objects, segmenting the related objects properly and/or classification of the objects in the image data. Feature inputs used for traditional machine learning methods are obtained by processing image data. In the CNN method, the image data feeds the network input layer directly. Features are acquired from these layers without using additional image processing methods. More data may be required to train the network comparing to traditional methods. On the other hand, the transfer learning approach can be utilized to retrain classification part of a pre-trained $\mathrm{CNN}$ model to speed-up the training phase.

In summary, the $\mathrm{CNN}$ approach can provide great flexibility and remarkable achievements in the classification of object images. In this context, different CNN models have been used to classify images of maize seeds as haploid and diploid.

Related studies are given in the second part of the study. In the third section, the dataset and recommended methods are mentioned. The experimental findings obtained on the dataset are given in the fourth section. In the fifth section, the results obtained within the scope of the study are discussed and future studies are mentioned. In the last part, the references for the study are given.

\section{Related Studies}

There are some prominent studies using computer-aided methods and approaches to facilitate the separation of maize seeds. Some studies have focused to identify seed characteristics by using special imagining equipment or software tools. Recently, a remarkable number of studies have investigated the machine learning (ML) methods to extract features of the maize seeds. Then these features are used to train classification algorithms based on ML. Moreover, electro-mechanical systems have been also developed to classify the maize seeds. Based on all these studies, literature have been deeply investigated and state-of-the-art studies have been analyzed.
Couto and Davide [9] aims to identify haploids by flow cytometry and relate nuclear DNA content to the morphological and morphometric properties of the seeds that reveal them. In the study, molecular markers have been used to confirm the androgenetic nature of haploid. Boote et al. [10] have developed a new fluorescentbased method for inbred haploid discrimination in maize kernels using R1-nj color marker with fluorescence microsproscopy and imaging. In the study, seven inbred lines have been used with the changing R1-nj marker. As a result of the experiments carried out; the fluorescent response of diploid nuclei at the embryonic spot point is shown to have a lower intensity. $\mathrm{Yu}$ et al. [11] have proposed a nonlinear feature analytical method to identify haploid maize seeds based on Supervised Virtual Sample Kernel Locality Preservation Projection to take full advantage of class label information. They have said that using nearinfrared spectroscopy technology to distinguish haploid seeds from hybrid seeds has the advantages of being non-destructive, fast, and cost-effective. Lin et al. [12] emphasized that maize haploid seeds have been automatically selectable using near-infrared (NIR) spectrum properties, thanks to the diffuse transmission technology of NIR spectroscopy. However, the NIR spectra of maize seeds have also been expressed to contain a lot of unnecessary features and noise that will reduce the identification performance. They have explained that they have overcome this problem by designing a low size and uniform size of the seed spectrum properties to improve the collected spectra. Wang et al. [13] aimed to investigate a fast and accurate method to identify haploid maize kernel using infrared hyperspectral imaging technology. This technology has been utilized to overcome the current automatic haploid identification limitations and provide a more accurate screening of haploid. Fuente et al. [14] focuses on defining the haploid lineage. In the study, six inbred lines have been developed with the maternal haploid inducer 'RWS / RWK-76' and a seed sample has been manually aligned for each line.

Wang et al. [15] have carried out the selection model design of high accuracy maize haploid seeds from diploid ones based on optimum waveband selection of the LSTM-CNN algorithm with deep learning and hyperspectral imaging technology. As a result of the experiments, it has been claimed that the accuracy rate reached $97 \%$ in the optimum waveband of 1367.6$1526.4 \mathrm{~nm}$. Altuntaş et al. [7] have proposed a method to classify haploid and diploid maize seeds using image processing and classification methods. Firstly, five different features are obtained from each maize seed image. Then the feature vectors are classified using a support vector machine classifier. In the study [16], texture features of maize seed embryos have been used. These features are derived from the gray level cooccurrence matrix. In another of their study [17], it is inspired by the latest achievements of deep transfer 
learning, they address this problem as a computer vision task to present a non-destructive, fast, and low-cost model. To achieve this goal, $\mathrm{CNN}$ have been used to automatically recognize haploid and diploid maize seeds with a transfer learning approach. Altuntaş and Kocamaz [18] have proposed a computer-based method for the identification of haploid maize seeds. Maize seed embryos have been segmented by the k-means clustering method. In RGB, HSV, and Lab color spaces, the first four-degree color moments have extracted for each color channel. The obtained features then have been classified with SVM.

Song et al. [19] have designed an automatic separation system that can separate the haploid maize seeds from hybrid seeds marked with the R1-nj label, in their study. The system includes seed feeding, image acquisition, sorting, and system control units. The seed feeding unit distributes the maize seeds on a synchronous belt. The image acquisition unit acquires images of maize seeds and separates the heterozygous from the haploid nuclei depending on the color property of the endosperm. Finally, the separation unit performs physical separation with mechanical arms and solenoid valves that can select the heterozygous seeds using air intake.

\section{Materials and Methods}

\subsection{Dataset}

The dataset consisting of 3000 maize seed images in total [17] contains 1230 haploid and 1770 diploid maize seed images. The dataset occupies 49.3 MB of disk space. Each image obtained is in RGB color space and format is JPEG. Since the resolutions of the images in the dataset vary between $300 \times 289$ pixels and $610 \times 637$ pixels depending on seeds size, the images have been resized to the appropriate resolution according to the input layer of each $\mathrm{CNN}$ model. In this context, while $227 \times 227$ resolution is used for the AlexNet model, $224 \times 224$ resolution is used for GoogLeNet, Resnet18, ResNet50, and VGG16 models. Sample maize seeds have been demonstrated in Figure 1.
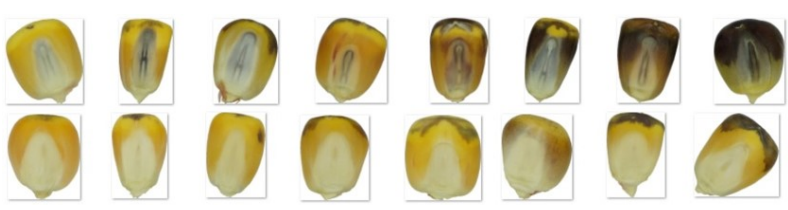

Figure 1. Sample diploid (top) and haploid (bottom) maize seed images from the dataset.

\subsection{Deep Feature Extraction}

A deep feature in the context of deep learning is that a unit (layer) within a hierarchical model responds consistently to an input, where this response contributes to the model's decision. Deep features are obtained from pre-trained $\mathrm{CNN}$ models. In other words, pre-trained CNN models can be used as feature extractors to feed any classifiers. Depending on where the model is located along with its hierarchical structure, one layer can be considered deeper than the other. Each of the network layers has deep features. Deep features are used in the network layers of the $\mathrm{CNN}$ model like convolution, pooling, etc. Their values/weights vary depending on the filter and mathematical operations applied in the layers. Deep features are expected to represent the highest level of images of objects intended to be classified.

AlexNet [20] is one of the pioneering deep learning algorithms. It has been introduced for the first time in 2012 as part of the ImageNet Large Scale Visual Recognition Challenge (ILSVRC). In this competition, it has attracted attention to the $\mathrm{CNN}$ architecture by providing a remarkable high performance. AlexNet is a CNN architecture with a total of 25 layers: input, convolution, ReLU (Rectified Linear Unit: it simply changes all the negative activations to 0 . It is a basic layer for all $\mathrm{CNN}$ architectures), normalization, pooling, fully connected layer, drop-out, classification, and output. The AlexNet model has a total of 60 million computational parameters. The architecture takes $227 \times 227$ colored images as input.

ResNet [21] is a CNN architecture that uses the residual learning framework module to facilitate network training. The focus of this architectural model is the degradation problem. The novelty of the model is the use of residual blocks and the depth in its architecture. In a conventional convolutional deep learning model, stacked layers fit a desired underlying mapping, while the ResNet model allows these layers to be seated in a residual mapping. In this study, Resnet-18 and ResNet50 have been used.

GoogLeNet [22] is another deep learning network that has managed to attract attention. Similarly, it has been revealed in the ILSVRC competition held in 2014. The most important difference in this architecture is the inception module added to the layers as a new block. This module consists of branching to more than one depth that works like a shortcut between layers. The most prominent feature of this CNN architecture is that the architecture increases width and depth while keeping the computational cost constant. It makes this possible with the newly added inception module. The module has improved the level of utilization of computing resources within the network architecture. GoogLeNet consists of 22 layers in total and 7 million computational parameters.

VGGNet [23] is a CNN architecture presented for the first time in the ILSVRC competition held in 2014. The most unique property about VGG16 is that instead of having many hyper-parameters, it is focused on having the convolution layers of a step-1 (stride-1) and $3 \times 3$ filter, and use always the same fill and maxpool layer of 
the $2 \times 2$ step- 2 (stride-2) filter. The convolution and maximum pool layers have been maintained consistently throughout the entire architecture. VGG16 is a fairly large network, it consists of 41 layers in total and has about 138 million (approximately) parameters.

\subsection{Classifier Methods}

A classification method uses a learning algorithm to define the model that is optimal to the relationship between the feature set of input data and the class label/tag. Therefore, the primary goal of the learning algorithm is to create a smart model that accurately predicts class tags of previously unknown records. The classifier method is employed to determine the label data of different classes in the utilized data set in this study.

Support Vector Machine (SVM) [24] are supervised learning models used for classification and regression analysis, analyzing data, with associated learning algorithms in machine learning. An SVM is a separator classifier that is formally defined by a separator hyperplane. In other words, when labeled training data is given, the algorithm produces an optimal hyperplane that categorizes new samples. The general SVM function dual version is given below (1). The secondorder (quadratic) SVM classifier has been used to avoid the local minimum, in the study.

$$
f(x)=\sum_{i=1}^{N} a_{i} \cdot y_{i} \cdot K\left(x, x_{i}\right)+b
$$

The $N$ value given in the equation indicates the number of support vectors. Parameter $K\left(x, x_{i}\right)$ expresses support vectors. The parameter $a$ refers to weight. The $y_{i}$ parameter indicates the class to which the observations belong. The $b$ corresponds to the bias rate threshold. The SVM model is the representation of samples as points in space so that samples of individual categories are divided into as wide space as possible. In addition to performing linear classification, SVMs can perform a nonlinear classification by indirectly matching their input to high-dimensional property fields. Given a set of training examples, each marked as one or the other of two classes, an SVM algorithm is used as a non-probable binary linear classifier by assigning new samples to one class or the other.

K-Nearest Neighbor (KNN) [25] is one of the basic classification algorithms in machine learning. Its classification approach is quite simple and can be easily applied to various problems. It is mainly based on feature similarity. KNN checks how similar a data point is to its neighbor and classifies the data point into the class it most closely resembles. In the experiments carried out in this study, Euclidean distance has been used for the KNN classifier. This distance calculation is made with equation (2).

$$
\sqrt{\sum_{i=1}^{k}\left(x_{i}-y_{i}\right)^{2}}
$$

The equation simply calculates the distance between the pairs $(x, y)$ in $2 \mathrm{~d}$ Cartesian space. Unlike most algorithms, $\mathrm{KNN}$ is a non-parametric model, meaning it makes no assumptions about the dataset. This makes the algorithm more effective as it can process realistic data. $\mathrm{KNN}$ is a lazy algorithm, which means that it memorizes the training dataset rather than learning a distinctive function from the training data. In this study, weighted KNN will be used as a classifier. Unlike the traditional $\mathrm{KNN}$ method, $K$ is weighted with the nearest neighbor $1 / k$ in weighted $\mathrm{KNN}$, while the weight of other data is considered to be 0 . For this purpose, the closest neighbor ' $i$.' is weighted for $\mathrm{n}$ samples with the following equation (3).

$$
\sum_{i=1}^{n} w_{n i}=1
$$

Decision Tree (DT) [26] is a simple and widely used classification technique. It applies a simple approach to solve the classification problem. The DT classifier performs a series of specially crafted branching about the features of the test data. If a result is reached about the class label of the data as a result of the branching, the algorithm is terminated; otherwise, the next branching continues until a result is reached. Within the scope of the study, the maximum deviation reduction (MDR) has been used as the division criterion. It is also known as the entropy function and found by the following equation (4).

$$
I_{E}(N)=-K \sum f(N, j) \log f(N, j)
$$

Here, $K$ is the proportion of observations, function $\boldsymbol{f}$ is the frequency of class $\boldsymbol{j}$ in node $\boldsymbol{N}$. The purpose of MDR is to reduce uncertainty until a pure leaf node is established. In DT, root and internal nodes contain feature test conditions to separate data with different features. All terminal nodes are assigned a class label 'Yes' or 'No'. Once DT is created, it is quite easy to classify a test record. Starting from the root node, the test condition is applied to the data and the appropriate branch is monitored according to the result of the test. The branching then takes place towards another inner node or a leaf node to which a new test condition is applied.

\section{Results}

The computer used in the experiments within the scope of the study has an I7 6700HQ CPU, 8GB RAM, 2GB GTX950 GPU, and 240GB SSD HDD. Experiments have been carried out using MATLAB (2019b) environment. 
The features of 3000 maize seed images have been obtained on the CNN models within the scope of the study. For this purpose, it is provided to extract features from 9 different layers in total over five different $\mathrm{CNN}$ models. Obtained features have been given to the classifier algorithms as input to perform the classification process. SVM, KNN, and DT classifiers have been used for this process. Regarding the classifiers used; in the DT classifier, the maximum split parameter (max split) value has been set to 20 . The partition criterion is made according to the MDR technique. In the KNN classifier; a weighted tree structure has been used and the $\mathrm{k}$ neighborhood parametric value is set to 20 . On the other hand, Euclidean distance has been used to measure neighborhood distance. The squared inverse technique is used as the weighting approach. As support vector classifier; the second-order (quadratic) SVM method has been used. The box constraint level is set to 1 . Kernel scaling has been used in auto mode. All these classifier adjustments have been determined empirically with different parametric combinations. The overall structure of the proposed model is shown in Figure 2.

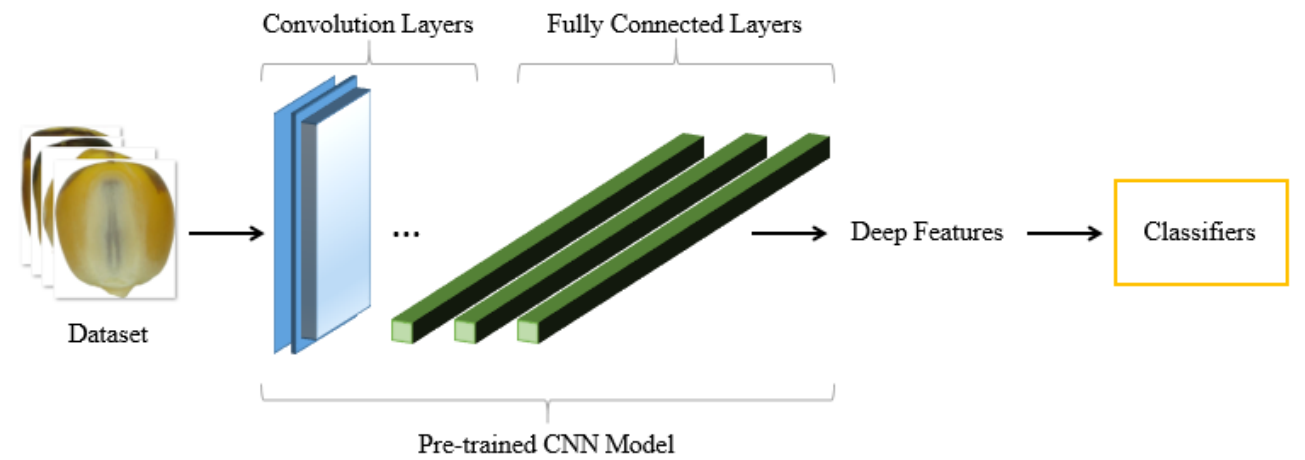

Figure 2. Overall structure of the proposed model.

In the AlexNet model feature extraction has been made from three layers: 'FC6', 'FC7', and 'FC8' layers. In the GoogLeNet model, features have been obtained from the fully connected layer named "Loos-3". In ResNet-18 and ResNet-50 models, the features have been obtained from the "FC1000" fully connected layers. Finally, in the VGG16 model, it has been provided to obtain features from 'FC6', 'FC7', and 'FC8' layers which are fully connected layers similar to the AlexNet model. The number of features obtained and the elapsed times of obtaining the features are given in Table 1 . The elapsed time of acquiring the features vary according to the number of layers of the models, processes like the applied mathematical filter, convolution and etc. In a low-complexity CNN model, features can be obtained more quickly.

Table 1. Feature acquiring times.

\begin{tabular}{lcc}
\hline CNN Model & $\begin{array}{c}\text { Feature } \\
\text { Number }\end{array}$ & $\begin{array}{c}\text { Feature Acquiring } \\
\text { Time }\end{array}$ \\
\hline AlexNet FC6 & 4096 & $382,86 \mathrm{~s}$ \\
AlexNet FC7 & 4096 & $422,20 \mathrm{~s}$ \\
AlexNet FC8 & 1000 & $212,50 \mathrm{~s}$ \\
GoogLeNet & 1000 & $332,08 \mathrm{~s}$ \\
ResNet18 & 1000 & $324,89 \mathrm{~s}$ \\
ResNet50 & 1000 & $310,17 \mathrm{~s}$ \\
VGG16 FC6 & 4096 & $1101,48 \mathrm{~s}$ \\
VGG16 FC7 & 4096 & $1121,74 \mathrm{~s}$ \\
VGG16 FC8 & 1000 & $958,94 \mathrm{~s}$ \\
\hline
\end{tabular}

In the study, 10-fold cross-validation method has been used to divide the dataset into training and test sets for obtain more generalized results. The effects of deep features obtained from fully connected layers on classification performance have been analyzed. Accuracy, precision, sensitivity, F-score, and classification times have been used as performance metrics to evaluate these effects. Accuracy, precision, sensitivity, and F-score performance metrics are calculated based on confusion matrix. The classification times refer to the time elapsing to classify the maize seed types according to the features.

The confusion matrix allows the measurement of model performance by comparing the number of true and false predictions made by classifiers on the test set for the CNN models. Since there are fewer haploid maize seeds compared to diploid maize seeds in this study, the positive class has been determined as haploids and the negative class has been determined as diploids. Among the samples labeled as haploid in the dataset, it is the true positive (TP) data that the proposed methods predict as haploid. What the method predicts as diploid is handled as false negative (FN). Among the samples labeled as diploid in the dataset, they are true negative (TN) data that the method predicts as diploid. Finally, the data estimated by the method as haploid have been evaluated as false positive (FP). Mathematical calculation formulas for performance metrics are as follows:

$$
\text { Accuracy }=\frac{T P+T N}{T P+F P+F N+T N}
$$




$$
\begin{gathered}
\text { Precision }=\frac{T N}{T N+F P} \\
\text { Sensitivity }=\frac{T P}{T P+F N} \\
F-\text { score }=\frac{2 T P}{2 T P+F P+F N}
\end{gathered}
$$

Feature classification experiment results performed with the SVM classifier are given in Table 2. The values given in the table are; confusion matrix (TP, FP, FN, and TN), accuracy (Acc.), precision (Prec.), sensitivity (Sens.), F-Score, and runtime of the SVM classifier. According to the acquired results, the $\mathrm{CNN}$ model with the highest performance of the SVM method is ResNet50 with an accuracy rate of $91.4 \%$. While the network with the second-highest performance is VGG16 with $91 \%$ accuracy, this performance has been achieved with the obtained features from the FC6 fully connected layer of the network. While the third-highest accuracy model is VGG16, 89.6\% accuracy has been achieved with the features acquired from the FC7 layer of this network. While the highest precision is 0.91 in the ResNet50 model, the highest sensitivity is in the VGG16 model with 0.93. The F1-Score used to eliminate extreme situations that occurred on the ResNet50 model has been determined by about 0.90 . When the classification working times of SVM classifier according to the obtained features from the models are examined; in layers where 1000 features are obtained, the elapsed time is less than 1 minute, while in layers where 4096 features are obtained, this time is over 2 minutes.

Table 2 Classifier Results with SVM Method.

\begin{tabular}{lccccccccc}
\hline CNN Model & TP & FP & FN & TN & Acc. & Pre. & Sen. & F-Score & Time \\
\hline AlexNet FC6 & 1120 & 110 & 214 & 1556 & $89,2 \%$ & 0,88 & 0,91 & 0,87 & $148,39 \mathrm{~s}$ \\
AlexNet FC7 & 1104 & 126 & 230 & 1540 & $88,1 \%$ & 0,87 & 0,90 & 0,86 & $154,82 \mathrm{~s}$ \\
AlexNet FC8 & 1083 & 147 & 255 & 1515 & $86,6 \%$ & 0,86 & 0,88 & 0,84 & $38,59 \mathrm{~s}$ \\
GoogLeNet $^{\text {ResNet18 }}{ }^{*}$ & 1076 & 154 & 254 & 1516 & $86,4 \%$ & 0,86 & 0,87 & 0,84 & $45,64 \mathrm{~s}$ \\
ResNet50 $^{1^{*}}$ & 1103 & 127 & 209 & 1561 & $88,8 \%$ & 0,88 & 0,90 & 0,87 & $32,04 \mathrm{~s}$ \\
VGG16 FC6 $^{2 *}$ & 1136 & 94 & 165 & 1605 & $\mathbf{9 1 , 4 \%}$ & 0,91 & 0,92 & 0,90 & $27,51 \mathrm{~s}$ \\
VGG16 FC7 $^{\text {VGG16 FC8 }}$ & 1139 & 91 & 178 & 1592 & $91,0 \%$ & 0,90 & 0,93 & 0,89 & $139,54 \mathrm{~s}$ \\
\hline
\end{tabular}

$* 1,2,3$; top three $\mathrm{CNN}$ models and layers with the highest performance

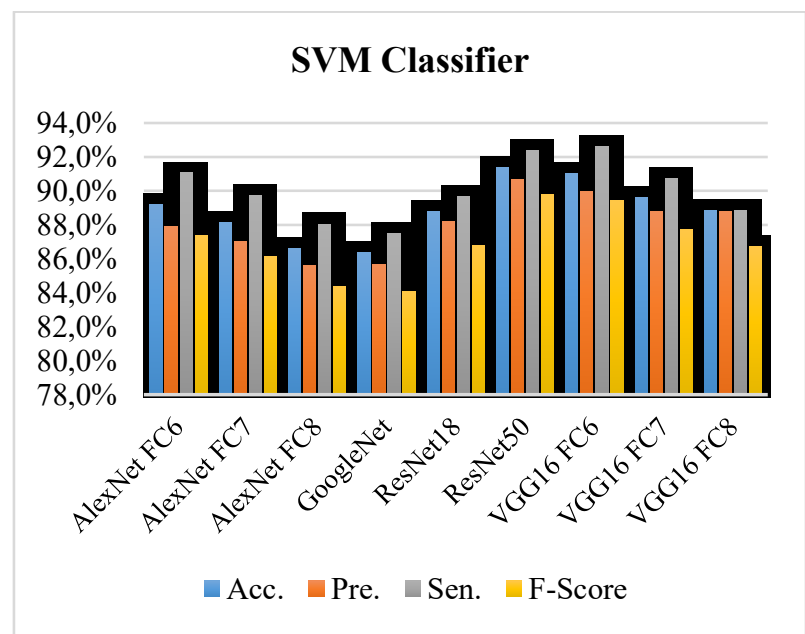

Figure 3. SVM classifier and performance metrics of CNN models.

Accuracy, precision, sensitivity, and F-Score values obtained in the pre-trained CNN models with the SVM classifier are given graphically in Figure 3.
Feature classification experiment results performed with the KNN classifier are given in Table 3. The highest performance has been obtained in the ResNet50 model with $87.5 \%$ accuracy in the classification experiments using the KNN classifier. The second-highest performance has been achieved in the VGG16 FC6 layer with an $84.9 \%$ accuracy rate, while the thirdhighest performance has been achieved in the ResNet18 model with $84.3 \%$ accuracy. The highest sensitivity value has been realized in the ResNet50 model with 0,84 , while the highest sensitivity is in the FC6 layer with 0,94 in the VGG16 model. The highest F-Score value has been obtained in the ResNet50 model with 0.86 . The acquired classification times in the KNN classifier are less than 1 minute for layers with 1000 features, and over 1 minute for layers with 4096 features. 
Table 3. Classifier Results with KNN Method.

\begin{tabular}{|c|c|c|c|c|c|c|c|c|c|}
\hline CNN Model & TP & $\mathbf{F P}$ & FN & $\mathbf{T N}$ & Acc. & Pre. & Sen. & F-Score & Time \\
\hline AlexNet FC6 & 1048 & 182 & 382 & 1388 & $81,2 \%$ & 0,78 & 0,85 & 0,79 & $92,07 \mathrm{~s}$ \\
\hline AlexNet FC7 & 1001 & 229 & 382 & 1388 & $79,6 \%$ & 0,78 & 0,81 & 0,77 & $83,75 \mathrm{~s}$ \\
\hline AlexNet FC8 & 955 & 275 & 379 & 1391 & $78,2 \%$ & 0,79 & 0,78 & 0,74 & $51,49 \mathrm{~s}$ \\
\hline GoogLeNet & 1021 & 209 & 360 & 1410 & $81,0 \%$ & 0,80 & 0,83 & 0,78 & $54,00 \mathrm{~s}$ \\
\hline ResNet18 $8^{3^{*}}$ & 1096 & 134 & 337 & 1433 & $84,3 \%$ & 0,81 & 0,89 & 0,82 & $51,99 \mathrm{~s}$ \\
\hline ResNet50 ${ }^{1^{*}}$ & 1130 & 100 & 276 & 1494 & $87,5 \%$ & 0,84 & 0,92 & 0,86 & $51,51 \mathrm{~s}$ \\
\hline VGG16 FC6 ${ }^{2^{*}}$ & 1162 & 68 & 385 & 1385 & $84,9 \%$ & 0,78 & 0,94 & 0,84 & $93,08 \mathrm{~s}$ \\
\hline VGG16 FC7 & 1106 & 124 & 384 & 1386 & $83,1 \%$ & 0,78 & 0,90 & 0,81 & $83,08 \mathrm{~s}$ \\
\hline VGG16 FC8 & 1104 & 126 & 371 & 1399 & $83,4 \%$ & 0,79 & 0,90 & 0,82 & $18,26 \mathrm{~s}$ \\
\hline
\end{tabular}

*1, 2, 3; top three CNN models and layers with the highest performance

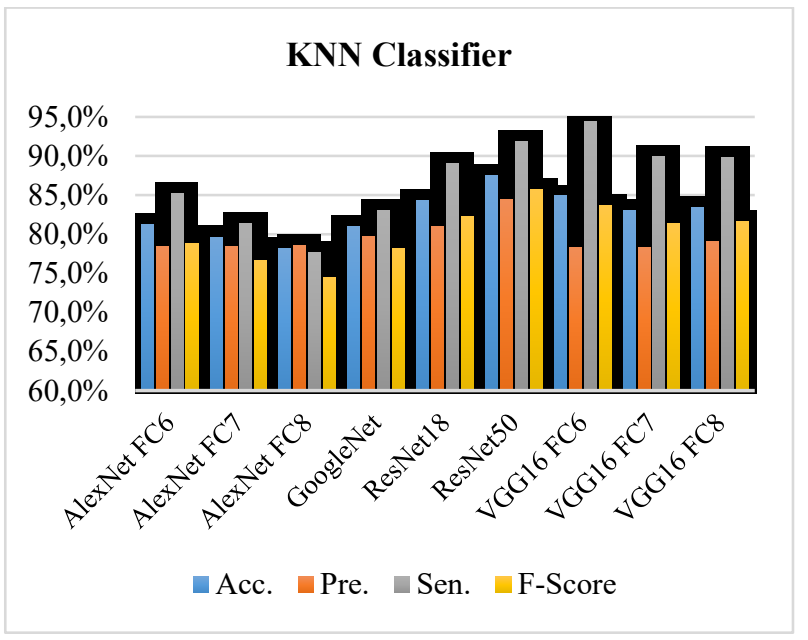

Figure 4. KNN classifier and performance metrics of CNN models.
Accuracy, precision, sensitivity, and F-Score values obtained through KNN classifier and pre-trained CNN models are graphically given in Figure 4.

Feature classification experiment results performed with the DT classifier are given in Table 4. The highest accuracy in the DT classifier is again in the ResNet50 model with a rate of $82.5 \%$. The second and third best accuracy rates have been obtained from ResNet18 and VGG16 FC6 models as $80.5 \%$ and $79.8 \%$, respectively. The best sensitivity value is realized in the ResNet50 model as 0.82 . The best sensitivity value has been found as 0.84 in the ResNet50 model. In F-Score values, the best value has been obtained as 0.80 with the ResNet50 model. When the obtained values according to the classification times of the DT classifier are examined; it has been observed that classifiers took less than 1 minute in all models. However, in layers containing 1000 features, this time is around 30 seconds at most, while in layers containing 4096 features, it is quite close to the

Table 4. Classifier Results with DT Method.

\begin{tabular}{lccccccccc}
\hline CNN Model & TP & FP & FN & TN & Acc. & Pre. & Sen. & F-Score & Time \\
\hline AlexNet FC6 & 894 & 336 & 407 & 1363 & $75,2 \%$ & 0,77 & 0,73 & 0,71 & $59,79 \mathrm{~s}$ \\
AlexNet FC7 & 958 & 272 & 429 & 1341 & $76,6 \%$ & 0,76 & 0,78 & 0,73 & $58,79 \mathrm{~s}$ \\
AlexNet FC8 & 920 & 310 & 430 & 1340 & $75,3 \%$ & 0,76 & 0,75 & 0,71 & $12,47 \mathrm{~s}$ \\
GoogLeNet $^{\text {ResNet18 }}{ }^{*}$ & 962 & 268 & 380 & 1390 & $78,4 \%$ & 0,79 & 0,78 & 0,75 & $31,47 \mathrm{~s}$ \\
ResNet50 $^{*}$ & 933 & 237 & 335 & 1435 & $80,5 \%$ & 0,81 & 0,80 & 0,77 & $30,97 \mathrm{~s}$ \\
VGG16 FC6 $^{*}$ & 1031 & 199 & 326 & 1444 & $\mathbf{8 2 , 5 \%}$ & 0,82 & 0,84 & 0,80 & $30,51 \mathrm{~s}$ \\
VGG16 FC7 $^{*}$ & 984 & 246 & 360 & 1410 & $79,8 \%$ & 0,80 & 0,80 & 0,76 & $57,84 \mathrm{~s}$ \\
VGG16 FC8 $^{*}$ & 954 & 276 & 373 & 1397 & $78,4 \%$ & 0,79 & 0,78 & 0,75 & $56,89 \mathrm{~s}$ \\
\hline
\end{tabular}

*1, 2, 3; top three CNN models and layers with the highest performance 


\section{Decision Tree Classifier}

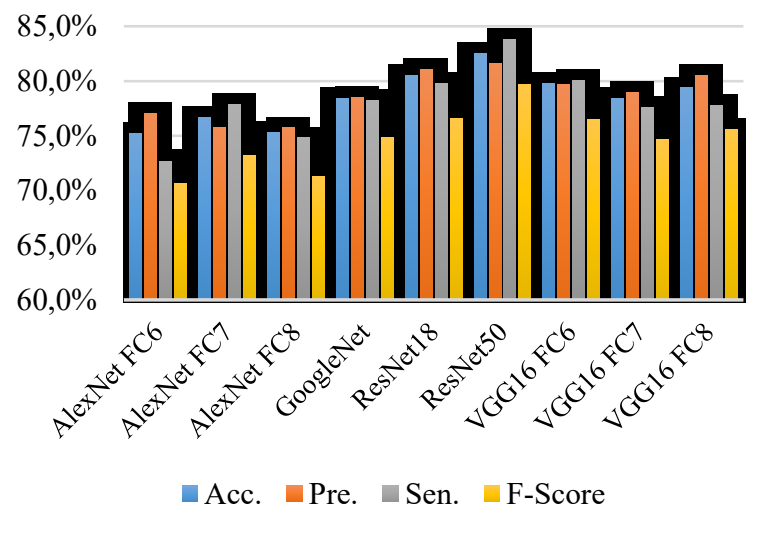

Figure 5. DT classifier and performance metrics of CNN models.

Accuracy, precision, sensitivity, and F-Score values obtained for the decision tree classifier and pre-trained $\mathrm{CNN}$ models are graphically given in Figure 5.

\section{Discussion and Conclusion}

In this study, including haploid and diploid seeds; features of 3000 maize seed images have been obtained from fully connected layers of pre-trained CNN models. Three different classifier methods have been proposed to classify maize seeds using these features. As a result of the experiments carried out, the highest accuracy in CNN models has been obtained in the ResNet50 model. While the best performance in precision (haploid recognition rate) values occurred in the ResNet50 model, the best model in sensitivity (diploid recognition rate) values is VGG16. In the VGG16 model, the layer with the highest value has been observed as the FC6 fully connected layer.

When the results are analyzed in terms of classification performance, the SVM classifier generally demonstrates the best classification performance in all CNN models. When using the SVM classifier, the highest accuracy has occurred in the ResNet50 model with $91.4 \%$, while the lowest performance is in the GoogLeNet model with $86.4 \%$. The second-best performance has been achieved with the KNN classifier. The highest performance with $\mathrm{KNN}$ is $87.5 \%$ in the ResNet50 model, while the lowest performance is $78.2 \%$ in the FC8 layer of the AlexNet model. DT classifier has shown the lowest classification performance. The highest achievement in accuracy with this classifier is again $82.5 \%$ in the ResNet50 model and the lowest has been observed in the AlexNet model FC6 layer with $75.2 \%$. This study successfully demonstrates the potential to perform computer-aided maize seed classification based on deep features obtained using pretrained CNN models. The study will be a source of inspiration for the systems that can be implemented in different types of agricultural products.
In future studies, in order to increase the performance, it is aimed to give embryo areas on the maize seed by segmenting them instead of giving all maize seed images as input directly to the models. On the other hand, based on the knowledge gained from this study, it is aimed to increase the performance to higher levels with the fusion of the features obtained from different $\mathrm{CNN}$ models. In other words, deep features from different CNN models will be used together. Fusion approaches will be developed to improve overall performance by readjusting these features.

\section{Author's Contributions}

Emrah Dönmez: Drafted and wrote the manuscript, proposed a CNN based classification approach for maize seeds. He has performed the experiment and analyzed the classification results.

\section{Ethics}

There are no ethical issues after the publication of this manuscript.

\section{References}

1. Altuntaş, Y, Kocamaz, AF, Yeroğlu, C. Identification of Apricot Varieties Using Leaf Characteristics and KNN Classifier. International Conference on Artificial Intelligence and Data Processing Symposium: IDAP-2019, 2019.

2. Dönmez, E, Zadeh, PV. A modified graph based approach for leaf segmentation with GPGPU support. 23rd Signal Processing and Communications Applications Conference: SIU-2015, 2015.

3. Chidzanga, C, Muzawazi, F, Midzi, J, Hove, T. 2017. Production and use of haploids and doubled haploid in maize breeding: A review. African J. Plant Breed; 201-213.

4. Prasanna, BM, Chaikam, V, Mahuku, G. 2012. Doubled haploid technology in maize breeding: theory and practice. CIMMYT.

5. Röber, FK, Gordillo, GA, Geiger, HH. 2005. In vivo haploid induction in maize - Performance of new inducers and significance of doubled haploid lines in hybrid breeding. Maydica.

6. Nanda, DK, Chase, SS. 1966. An Embryo Marker for Detecting Monoploids of Maize (Zea Mays L.1). Crop Sci; 6: 213-215.

7. Altuntaş, $\mathrm{Y}$, Kocamaz, AF, Cengiz, R, Esmeray, M. Classification of haploid and diploid maize seeds by using image processing techniques and support vector machines. 26th IEEE Signal Processing and Communications Applications Conference: SIU2018, 2018 .

8. Lecun, Y, Bengio, Y, Hinton, G. 2015. Deep learning. Nature; 521: 436-444

9. Couto, EG de O, Davide, LMC, Bustamante, F de O, Von, Pinho $\mathrm{RG}$, Silva, TN. 2013. Identification of haploid maize by flow cytometry, morphological and molecular markers. Ciência $e$ Agrotecnologia.

10. Boote, BW, Freppon, DJ, Fuente, GN de La, Lübberstedt, T, Nikolau, BJ, Smith, EA. 2016. Haploid differentiation in maize kernels based on fluorescence imaging. Plant Breed. 
11. Yu, L, Liu, W, Li, W, Qin, H, Xu, J, Zuo, M. 2018. Nondestructive identification of maize haploid seeds using nonlinear analysis method based on their near-infrared spectra Biosyst. Eng.

12. Lin, J, Yu, L, Li, W, Qin, H. 2018. Method for Identifying Maize Haploid Seeds by Applying Diffuse Transmission Near-Infrared Spectroscopy. Appl. Spectrosc; 72: 611-617.

13. Wang, Y. et al. 2018. Identification of maize haploid kernels based on hyperspectral imaging technology. Comput. Electron. Agric.

14. Fuente, GN de La, Carstensen, JM, Edberg, MA, Lübberstedt, T 2017. Discrimination of haploid and diploid maize kernels via multispectral imaging. Plant Breed.

15. Wang, XY, Liao, WX, An, D, Wei, Y. 2018. Maize Haploid Identification via LSTM-CNN and Hyperspectral Imaging Technology. CoRR; abs/1805.0.

16. Altuntaş, Y, Kocamaz, AF, Cömert, Z, Cengiz, R, Esmeray, M. Identification of Haploid Maize Seeds using Gray Level Cooccurrence Matrix and Machine Learning Techniques. International Conference on Artificial Intelligence and Data Processing, IDAP2018, 2019

17. Altuntas, Y, Cömert, Z, Kocamaz, AF. 2019. Identification of haploid and diploid maize seeds using convolutional neural networks and a transfer learning approach. Comput. Electron. Agric.

18. Altuntas, Y, Kocamaz, AF. 2019. Renk Momentleri ve Destek Vektör Makineleri Kullanarak Haploid Misır Tohumlarının Tanımlanmasında Renk Uzaylarının Sınıflandırma Performansına
Etkisinin Karşılaştırılması. Fırat Üniversitesi Mühendislik Bilim. Derg; 31: 551-560.

19. Song, $\mathrm{P}$, Zhang, H, Wang, C, Luo, B, Zhang, JX. 2018. Design and Experiment of a Sorting System for Haploid Maize Kernel. Int. J. Pattern Recognit. Artif. Intell.

20. Krizhevsky, A, Sutskever, I, Hinton, GE. 2012. ImageNet classification with deep convolutional neural networks. Advances in Neural Information Processing Systems.

21. He, K, Sun, J. Deep Residual Learning for Image Recognition, IEEE Conference on Computer Vision and Pattern Recognition (CVPR), 770-778, 2016.

22. Szegedy, C. et al. Going deeper with convolutions, Proceedings of the IEEE Computer Society Conference on Computer Vision and Pattern Recognition, 2015.

23. Simonyan, K. Zisserman, A. Very deep convolutional networks for large-scale image recognition, 3rd International Conference on Learning Representations: ICLR-2015, Conference Track Proceedings, 1-14, 2015.

24. Zanaty, EA. 2012. Support Vector Machines (SVMs) versus Multilayer Perception (MLP) in data classification. Egypt. Informatics $J$.

25. Guo, G., Wang, H, Bell, D, Bi, Y, Greer, K. 2013. KNN modelbased approach in classification. Lect. Notes Comput. Sci.

26. Kotsiantis, SB. Decision trees: A recent overview. Artificial Intelligence Review. 